\title{
A Systematic Algorithm for the Design of Hybrid Waveguide-Microstrip Transversal Microwave Filters
}

\author{
Mónica Martínez-Mendoza, Juan Sebastián Gómez-Díaz, David Cañete-Rebenaque, \\ Alejandro Alvarez-Melcon, and Smain Amari,
}

Technical University of Cartagena, Cuartel de Antigones s/n, Cartagena, ES-30202, Spain, E-mail: shanaz00@hotmail.com

January 10, 2011

Key Words: Band-pass filters, resonator filters, transversal filters, synthesis techniques, hybrid technology

\begin{abstract}
A novel algorithm to design hybrid waveguide-microstrip transversal microwave filters is proposed in this paper. The idea consists in isolating each resonator involved in the filter structure, to synthesize individually its frequency response, both in resonant frequency and bandwidth. These separated optimizations lead to the dimensions of the filtering structure to meet the requirements of a certain, specified transfer function. The novel algorithm introduces an iterative procedure in order to synthesize the direct coupling term between source and load, which is always present in the hybrid filter structure. The whole algorithm is illustrated by means of a design example, where a very good agreement between the ideal specified transfer function and the final frequency response of the designed hybrid filter is obtained. A second different design example, which has been manufactured and tested, is also included for validation.
\end{abstract}

\section{Introduction}

The development of accurate algorithms to design microwave filters with transmission zeros located at precise frequencies, is important to satisfy the increasing requirements of the current communication systems. This is one of the important steps before the physical implementation of the filter. However, the whole process to implement a specific filter is more complicated and requires several stages.

First of all, a specific technology must be chosen according to the appropriate characteristics of a particular application. Second, we have to fix a suitable coupling configuration that our technology can easily implement. Once this is done, we need to find the associated coupling matrix to represent the required amount of coupling between the different parts of the filter. This is usually done with analytical techniques able to synthesize specific frequency responses. To carry out this stage, 
the $(N+2)$ coupling matrix can be used with the process reported for the first time in [1]. Other analytical techniques can also be used for specific coupling topologies, as for instance the one reported in [2]. An example of a second order bandpass transversal filter based on the use of simple inductive discontinuities in a rectangular cavity was introduced in [3]. Two bandpass transversal filters implemented in simple microstrip structures were also presented in [4]. More recently, new transversal filters of second and third orders have been proposed using a hybrid waveguide-microstrip technology [5], [6]. Following this idea, one of the resonances of the filter is implemented with a cavity resonance, while the others are implemented using standard printed line microstrip resonators. A basic hybrid structure for a second order transversal filter can be seen in Fig. 1.

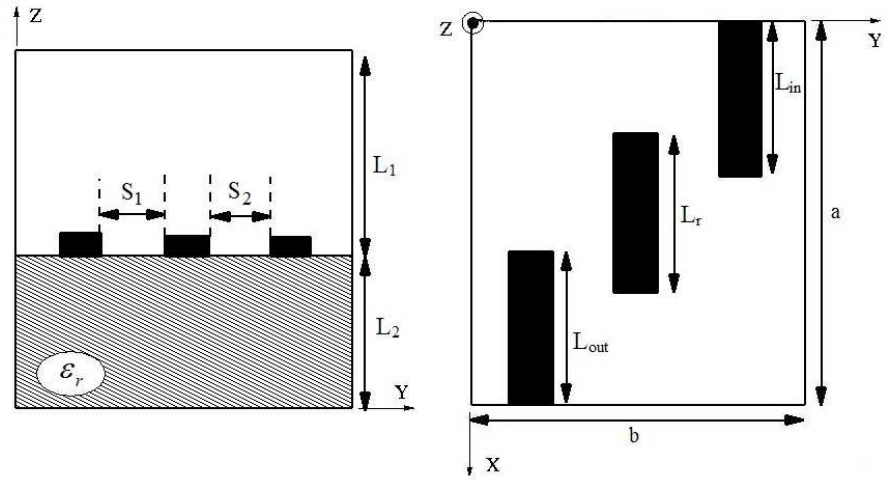

Figure 1: Hybrid waveguide-microstrip structure for a second order transversal filter. From $[5]$.

However, before we can obtain the final filter in any of these technologies, we will have to obtain the appropriate dimensions of the geometry, starting with the obtained coupling matrix. This last step can be quite difficult, as we have to find the relationship between the coupling terms of the matrix and the real dimensions of the structure. Several investigations, based on optimization techniques $[7,8,9]$, have been undertaken to solve this problem. While these optimization techniques are in general effective, they have convergence difficulties when the complexity of the structure increases. In these situations very long optimization times may be required.

An alternative approach to solve the design problem was introduced in [10]. This procedure is based on the decomposition of the design task into several simpler tasks, avoiding the use of optimization techniques directly on the overall complex structure. Following this technique, each resonator is extracted from the whole network, and then it is optimized sequentially. This avoids the need to optimize a large set of geometrical parameters at the same time.

This contribution is focused on the development of a filter design technique suitable for the new hybrid waveguide-microstrip technology proposed in [5]. The technique is based on the above mentioned idea of separating the design task into several simpler tasks. The main novelty with respect to the work originally developed in [10], is that the idea is now applied to the design of transversal filters, instead of in-line filters. Another novel aspect of this paper is that an iterative procedure is proposed, in order to adjust the direct coupling from the input to the output port, which is always present in this new hybrid technology. A design example is also included to show the validity of the technique. 


\section{Theoretical Review}

In this section, we are going to review the basic principles that will allow us to carry out the design procedure, using the filter topology shown in Fig. 1. It can be seen that it corresponds to a second order transversal filter using the hybrid waveguide-microstrip technology proposed in [5]. The $(N+2)$ fully canonical coupling matrix of a second order bandpass transversal filter, is known to be of the form shown in Table 1. The basic idea of the technique is to isolate the behavior of each

Table 1: Coupling matrix of a second order bandpass transversal filter.

\begin{tabular}{|c|cccc|}
\cline { 2 - 5 } \multicolumn{1}{c|}{} & $\mathrm{S}$ & 1 & 2 & $\mathrm{~L}$ \\
\hline $\mathrm{S}$ & 0 & $M_{S 1}$ & $M_{S 2}$ & $M_{S L}$ \\
1 & $M_{S 1}$ & $M_{11}$ & 0 & $M_{1 L}$ \\
2 & $M_{S 2}$ & 0 & $M_{22}$ & $M_{2 L}$ \\
$\mathrm{~L}$ & $M_{S L}$ & $M_{1 L}$ & $M_{2 L}$ & 0 \\
\hline
\end{tabular}

resonator. From the coupling matrix shown in Table 1, we can extract the behavior of a general $i$-th resonator $(i=1,2)$, using the coupling matrix shown in Table 2. In this coupling matrix, the diagonal

Table 2: Coupling matrix of the isolated $i$-th resonator $(i=1,2)$.

\begin{tabular}{|c|ccc|}
\cline { 2 - 4 } \multicolumn{1}{c|}{} & $\mathrm{S}$ & $\mathrm{i}$ & $\mathrm{L}$ \\
\hline $\mathrm{S}$ & 0 & $M_{S i}$ & 0 \\
$\mathrm{i}$ & $M_{S i}$ & $M_{i i}$ & $M_{i L}$ \\
$\mathrm{~L}$ & 0 & $M_{i L}$ & 0 \\
\hline
\end{tabular}

element $M_{i i}$ accounts for the difference in the resonant frequency of the resonator, with respect to the center frequency of the filter. This term of the coupling matrix is always different from zero in asynchronously tuned filters. Specifically, if the diagonal term is negative, the resonant frequency of that resonator will be higher than the center frequency of the filter. If on the contrary, the diagonal term is positive, then the resonant frequency of that resonator will be lower than the center frequency of the filter. Thus, if the following lowpass to bandpass transformation is applied:

$$
\omega=\frac{f_{c}}{\Delta x}\left(\frac{f}{f_{c}}-\frac{f_{c}}{f}\right)
$$

then the resonant frequency of the isolated resonator can be easily obtained. In above equation, $f$ is the frequency, $f_{c}$ is the center frequency of the passband, $\Delta x$ is the bandwidth and $\omega$ is the normalized frequency. Extracting the frequency $(f)$ from equation (1), we obtain:

$$
f=\frac{1}{2}\left[\omega \Delta x+\sqrt{\omega^{2} \Delta x^{2}+4 f_{c}^{2}}\right]
$$

Now, $(\omega)$ can be substituted by the corresponding diagonal element with its sign changed $\left(-M_{i i}\right)$. This is because, as already said, the shift of the resonant frequency is to the left if the sign is positive, and to the right if the sign is negative. In this way, the frequency obtained will be directly the resonant frequency of the resonator under study, namely:

$$
f_{R i}=\frac{1}{2}\left[-M_{i i} \Delta x+\sqrt{\left(-M_{i i}\right)^{2} \Delta x^{2}+4 f_{c}^{2}}\right]
$$


In the coupling matrix shown in Table 2 , the elements $\left(M_{S i}=M_{L i}\right)$ accounts for the coupling between the input and output ports to the corresponding $i$-th resonator. We know that this entry is related to the impedance inverter in the equivalent electrical network, or to the $3 \mathrm{~dB}$ bandwidth of the resonator in its physical response. The normalized $3 \mathrm{~dB}$ bandwidth of the resonator can be expressed as a function of the $3 \mathrm{~dB}$ bandwidth in the final transformed frequency axis. To do so, the normalized $3 \mathrm{~dB}$ bandwidth is defined as:

$$
\Delta \omega_{3 d B}=\omega_{2}-\omega_{1}
$$

Applying the lowpass to bandpass transformation to the normalized cut-off frequencies, we obtain:

$$
\Delta \omega_{3 d B}=\frac{f_{c}}{\Delta x}\left(\frac{f_{2}}{f_{c}}-\frac{f_{c}}{f_{2}}\right)-\frac{f_{c}}{\Delta x}\left(\frac{f_{1}}{f_{c}}-\frac{f_{c}}{f_{1}}\right)
$$

By straightforward transformations, this expression results into:

$$
\Delta \omega_{3 d B}=\frac{f_{c}}{\Delta x}\left(f_{2}-f_{1}\right)\left(\frac{1}{f_{c}}+\frac{f_{c}}{f_{2} f_{1}}\right)
$$

Furthermore, by definition, the $3 \mathrm{~dB}$ bandwidth in the final frequency axis is: $\Delta f_{3 d B}=f_{2}-f_{1}$, so we can write:

$$
\Delta \omega_{3 d B}=\frac{f_{c}}{\Delta x} \Delta f_{3 d B}\left(\frac{1}{f_{c}}+\frac{f_{c}}{f_{2} f_{1}}\right)
$$

Besides, the center frequency of the filter can be defined as the geometrical mean with respect to the two cut-off frequencies, for narrow bandpass filters: $f_{c}=\sqrt{f_{2} f_{1}}$. Thus, equation (7) results into:

$$
\Delta \omega_{3 d B}=2 \frac{\Delta f_{3 d B}}{\Delta x}
$$

On the other hand, it can be found in [11] that the scaled external quality factor is:

$$
q_{e}=Q_{e} F B W
$$

where the quality factor $Q_{e}$ is given by [12]:

$$
Q_{e}=\frac{2 \omega_{0}}{\Delta \omega_{3 d B}}
$$

and the fractional bandwidth $(F B W)$ is defined as:

$$
F B W=\frac{\Delta \omega}{\omega_{0}}
$$

The equiripple bandwidth $\Delta \omega$ in the lowpass prototype is equal to $\Delta \omega=2$, as it ranges from -1 $\mathrm{rad} / \mathrm{sec}$ to $+1 \mathrm{rad} / \mathrm{sec}$. Thus, the scaled external quality factor can be expanded as:

$$
q_{e}=\frac{2 \omega_{0}}{\Delta \omega_{3 d B}} \frac{\Delta \omega}{\omega_{0}}=\frac{4}{\Delta \omega_{3 d B}}
$$

Besides, it can also be found in [11] that the coupling between the input/output port and each resonator $\left(M_{S i}\right)$, and the scaled external quality factor are related as follows:

$$
M_{S i}^{2}=\frac{1}{q_{e}}
$$

Substituting equation (8) into (12), and the result into (13), we can find the final relation between the coupling term $M_{S i}$ and the $3 \mathrm{~dB}$ bandwidth of the resonator in the final transformed frequency axis:

$$
\Delta f_{3 d B}=2 \Delta x M_{S i}^{2}
$$

At this point, beginning with the coupling matrix of an isolated resonator, which is shown in Table 2, we can find the $3 \mathrm{~dB}$ bandwidth of the resonator response in the final transformed frequency axis $\left(\Delta f_{3 d B}\right)$, and also its resonant frequency $\left(f_{R i}\right)$, by using equations $(3)$ and (14). They will be used in the next section for the design of practical filters using the hybrid waveguide-microstrip technology. 


\section{Design Procedure}

The algorithm we are going to introduce, will allow us to design a transversal microwave filter in hybrid waveguide-microstrip technology with a fixed frequency response. This hybrid filter was first introduced in [5]. It consists of the compact structure shown in Fig. 1, which combines in a unique filter two different technologies, the waveguide and the microstrip, to achieve a second order bandpass, transversal, fully canonical design.

It is important to note that this structure implements the modified doublet topology, first introduced in [13]. This means that the coupling matrix will have one of the four couplings negative. In the case of the hybrid structure this is implemented by the change in sign produced in the components of the electromagnetic field that are responsible for coupling to the feeding lines (see [5]). This cavity mode behaves as one of the resonators, whereas the other is implemented by the open line microstrip resonator in the printed circuit. Besides, it was stated in the original work that this structure will always have a direct coupling from input to the output port. This makes possible to obtain fully canonical responses for maximum selectivity $\left(M_{S L} \neq 0\right)$.

The design procedure exploits the basic principles of the idea introduced in [10], but applied to the transversal hybrid structure. The flow chart of the design algorithm, that will be detailed in the following, is shown in Fig. 2 for a better understanding.

To illustrate the use of the algorithm with a real example, we are going to design a bandpass filter with two transmission zeros symmetrically placed at each side of the passband. The filter specifications are listed in Table 3.

Table 3: Filter specifications. First design

\begin{tabular}{|c|c|}
\hline Centre frequency & $4 \mathrm{GHz}$ \\
\hline Bandwidth $\Delta x$ & $70 \mathrm{MHz}$ \\
\hline Return Loss & $15 \mathrm{~dB}$ \\
\hline Transmission Zeros & $3.55 \mathrm{GHz} \& 4.45 \mathrm{GHz}$ \\
\hline
\end{tabular}

First, as indicated in Fig. 2, the value of the relative permittivity of the substrate and the cavity length will be fixed to $\epsilon_{r}=2$ and $a=40 \mathrm{~mm}$ respectively. Moreover, the width of the microstrip line and the input/output ports will be fixed to $2 \mathrm{~mm}$ during the whole design process.

Next, the direct synthesis procedure of [1] is used to obtain the $(N+2)$ transversal coupling matrix:

$$
M_{a}=\left(\begin{array}{rrrr}
0 & 0.7259 & -0.7342 & 0.0083 \\
0.7259 & -1.2908 & 0 & 0.7259 \\
-0.7342 & 0 & 1.2866 & 0.7342 \\
0.0083 & 0.7259 & 0.7342 & 0
\end{array}\right)
$$

According to Fig. 2, the next step is the identification of resonators. In matrix $M_{a}$, the resonator that does not change sign is tuned above the center frequency of the filter $\left(M_{11}\right.$ is negative and 


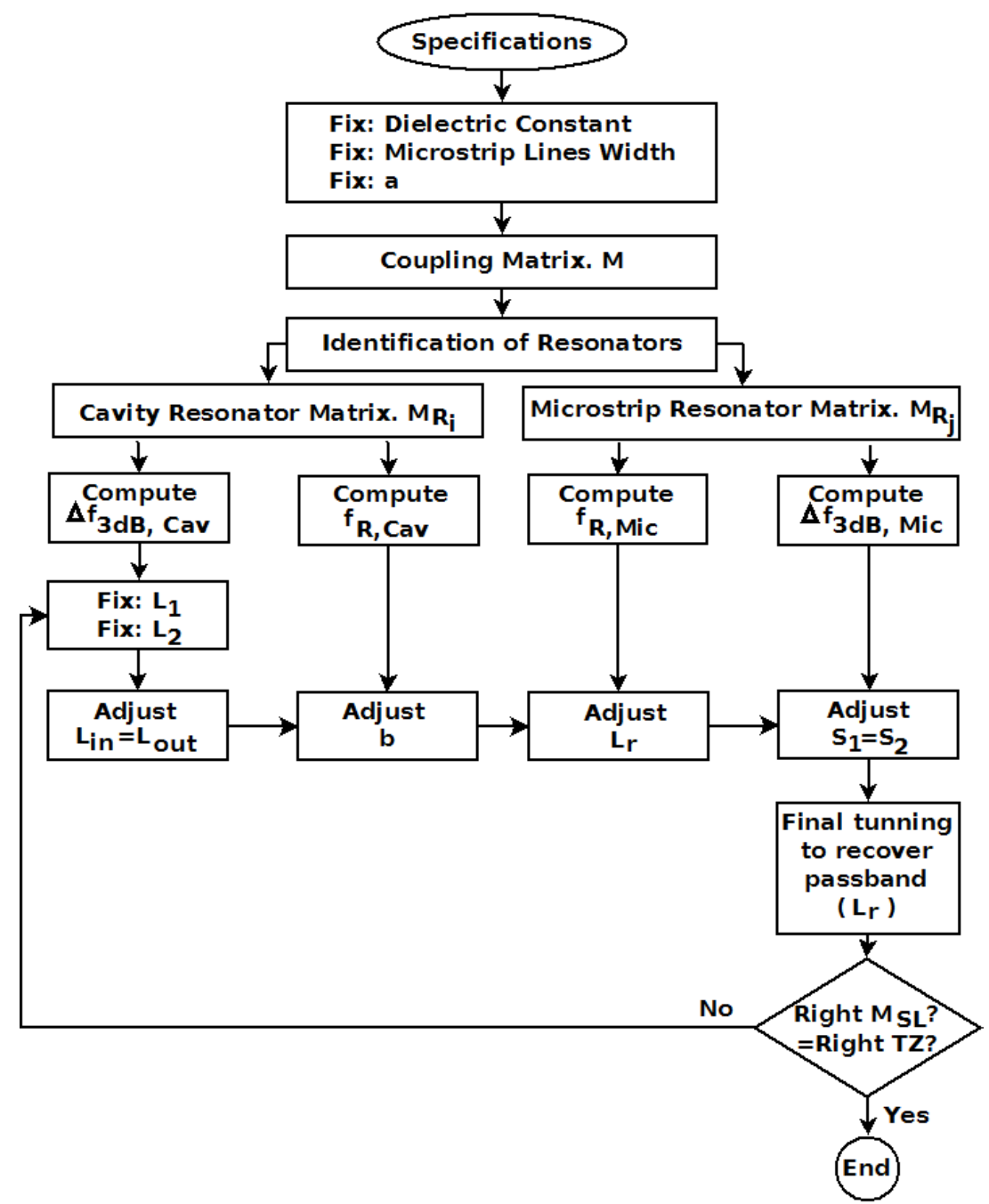

Figure 2: Flow chart of the design algorithm for the design of hybrid waveguide-microstrip transversal filters.

$\left.M_{S 1}=M_{L 1}\right)$. Moreover, the resonator changing sign from input to output is tuned below the center frequency of the filter $\left(M_{22}\right.$ is positive and $\left.M_{S 2}=-M_{L 2}\right)$. So, we already know that the resonator $(i=1)$ with higher resonant frequency will be implemented by the printed line microstrip resonator, whereas the resonator $(i=2)$ with lower resonant frequency will be implemented by the cavity mode.

The next step in the design procedure is the isolation of the individual resonator coupling matrixes. This will allow to calculate two parameters for each resonator: the resonant frequency and the $3 \mathrm{~dB}$ bandwidth in the final transformed frequency axis. In our example design, the first resonator is isolated with the following coupling matrix:

$$
M_{R 1}=\left(\begin{array}{rrr}
0 & 0.7259 & 0 \\
0.7259 & -1.2908 & 0.7259 \\
0 & 0.7259 & 0
\end{array}\right)
$$

Thus, applying equations (3) and (14), the required parameters can be straightforwardly determined as:

$$
f_{R 1}=4.0454 \mathrm{GHz}, \quad \Delta \mathrm{f}_{3 \mathrm{~dB}, \mathrm{R} 1}=73.765 \mathrm{MHz}
$$

On the other hand, the second resonator, implemented by the cavity mode, is associated with the 
following coupling matrix:

$$
M_{R 2}=\left(\begin{array}{rrr}
0 & -0.7342 & 0 \\
-0.7342 & 1.2866 & 0.7342 \\
0 & 0.7342 & 0
\end{array}\right)
$$

By using again equations (3) and (14), the resonant frequency and the $3 \mathrm{~dB}$ bandwidth in the final transformed frequency axis of the second resonator are found to be:

$$
f_{R 2}=3.9552 \mathrm{GHz}, \quad \Delta \mathrm{f}_{3 \mathrm{~dB}, \mathrm{R} 2}=75.475 \mathrm{MHz}
$$

Now that we have characterized each resonator in the filter, we can optimize them separately, until the right dimensions are obtained. We are going to illustrate the whole process in our example of Table 3. First, we fix the initial values $L_{1}=2.8 \mathrm{~mm}$ and $L_{2}=2.7 \mathrm{~mm}$. Once these dimensions have been fixed, the optimization process begins.

First, we look for the required frequency response of the cavity mode resonator. To do this, we eliminate the printed line microstrip resonator in Fig. 1. The first step is to adjust the input/output port lengths $L_{\text {in }}=L_{\text {out }}$ to achieve the required $3 \mathrm{~dB}$ bandwidth in the final transformed frequency axis. To aid in the adjustment of this resonator we can produce the graphic shown in Fig. 3 . The graphic plots the $3 \mathrm{~dB}$ bandwidth that can be achieved, as a function of the input/output ports length, and for different values of the substrate thickness $\left(L_{2}\right)$. In the same graphic we plot in the right $y$ axis, the relation between the amount of coupling $\left(M_{S 2}\right)$, and the required bandwidth, according to equation (14). The dashed line with arrows indicates that the coupling $M_{S 2}=0.7342$ requires

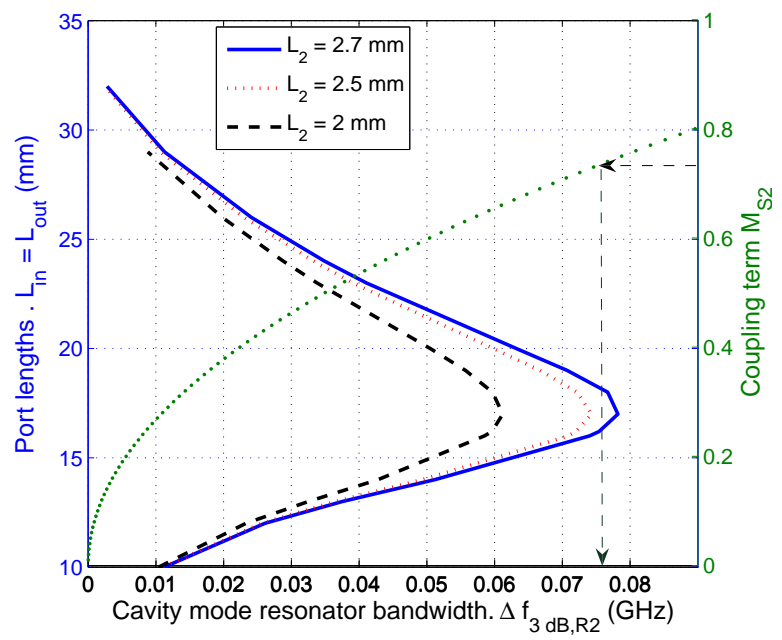

Figure 3: Cavity mode resonator bandwidth as a function of the port lengths (left $y$-axis), and of the coupling term $M_{S 2}$ (right $y$-axis).

a bandwidth of $\Delta f_{3 d B, R 2}=75.475 \mathrm{MHz}$. In the same graphic we can obtain, using the left $y$-axis, the required input/output port length needed to implement this bandwidth. This parametric study is very illustrative, and indicates that the required bandwidth of this design cannot be obtained for substrate thickness smaller than $L_{2}=2.5 \mathrm{~mm}$. The graphic is also useful since it aids in the selection of appropriate initial values for $L_{2}$ (in the example we have selected an initial value of $L_{2}=2.7 \mathrm{~mm}$ ). The left $y$-axis in this graphic indicates that a value $L_{i n}=L_{\text {out }}=16.2 \mathrm{~mm}$ is needed to synthesize the required $M_{S 2}$ coupling $\left(\Delta f_{3 d B, R 2}=75.475 \mathrm{MHz}\right)$. 
The next step consists in finding the waveguide width $(b)$ which provides the right resonant frequency. To aid in this task we can produce a graphic of the type shown in Fig. 4 . The graphic

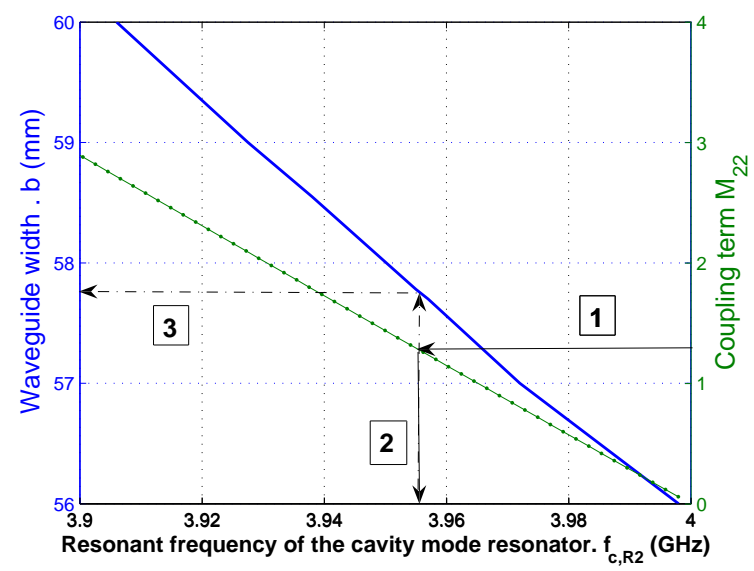

Figure 4: Resonant frequency of the cavity mode resonator as a function of the waveguide width (b) (left $y$-axis), and of the diagonal term $M_{22}$ (right $y$-axis).

shows the resonant frequency of the resonator as a function of the waveguide width (b) shown in Fig. 1. In the right $y$-axis of the same graphic we show the relation between the diagonal term $M_{22}$, and the required resonant frequency of the resonator, according to equation (3). Using this right $y$-axis, we can compute the required resonant frequency corresponding to the diagonal term $M_{22}=1.2866$ (see labels 1 and 2 in Fig. 4). In this case, the resonant frequency is $f_{R 2}=3.9552 \mathrm{GHz}$. Next, we have to determine the waveguide width $(b)$ which implements this resonant frequency in the filter structure. This can easily be done using the left $y$-axis of Fig. 4 (see labels 2 and 3 in the graphic). From the graphic, the waveguide width obtained is $b=57.70 \mathrm{~mm}$.

High accuracy in the synthesized frequency response for the cavity resonator, both in resonant frequency and in bandwidth, is obtained. Once the cavity resonator has been synthesized, the next step is to look for the required frequency response of the printed line microstrip resonator. To do this, we add the central microstrip line resonator as shown in Fig. 1. Before we can proceed with the synthesis of this resonator, we need to detune the cavity resonator. In this way the cavity resonance will not interfere with the microstrip resonance during the synthesis procedure. To detune the cavity resonator, we can simply take a smaller cavity width to send its resonant frequency to higher values (for instance, we take $b=40.5 \mathrm{~mm}$ in our example).

To aid in the adjustment of the resonant frequency of the printed microstrip resonator, we can produce the graphic shown in Fig. 5. The graphic shows the resonant frequency of the printed line as a function of its length ( $L_{r}$ in Fig. 1). Also, in the right $y$-axis of the same graphic we plot the relation between the diagonal term $M_{11}$, and the required resonant frequency of the resonator. Using first this right $y$-axis, we can compute the resonant frequency corresponding to the diagonal term $M_{11}=-1.2908$ (see labels 1 and 2 in Fig. 5). In this way, we find the resonant frequency $f_{R 1}=4.0454 \mathrm{GHz}$. Next, using the left $y$-axis of the same graphic, we find the required length of the printed line to obtain this resonant frequency (see labels 2 and 3 in Fig. 5). Following this procedure we obtain the length of the printed line resonator as $L_{r}=28.73 \mathrm{~mm}$.

Once the line length has been determined, we have to optimize the air gaps $\left(S_{1}\right.$ and $S_{2}$ in Fig. 1) 


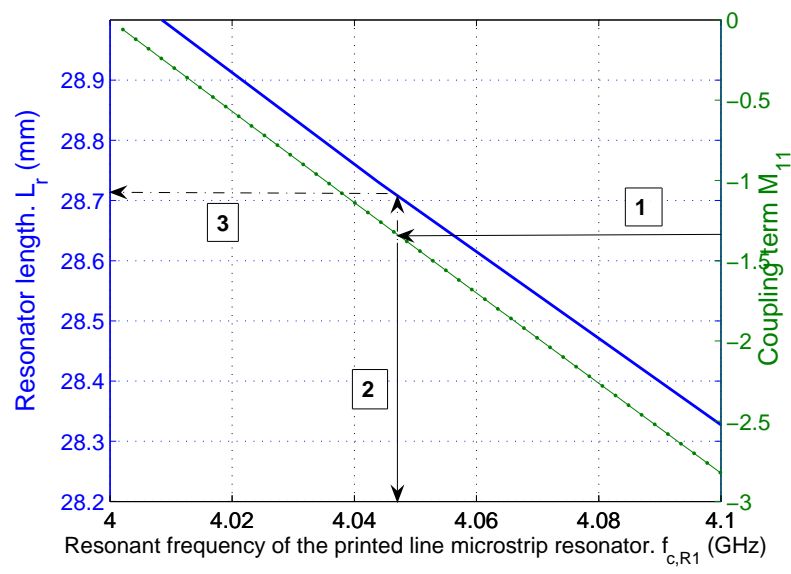

Figure 5: Resonant frequency of the printed line microstrip resonator as a function of the line length (left $y$-axis), and of the diagonal term $M_{11}$ (right $y$-axis).

to obtain the required $3 \mathrm{~dB}$ bandwidth in the final transformed frequency axis. To aid in this task we can produce the graphic shown in Fig. 6 . In this graphic we present the $3 \mathrm{~dB}$ bandwidth of the

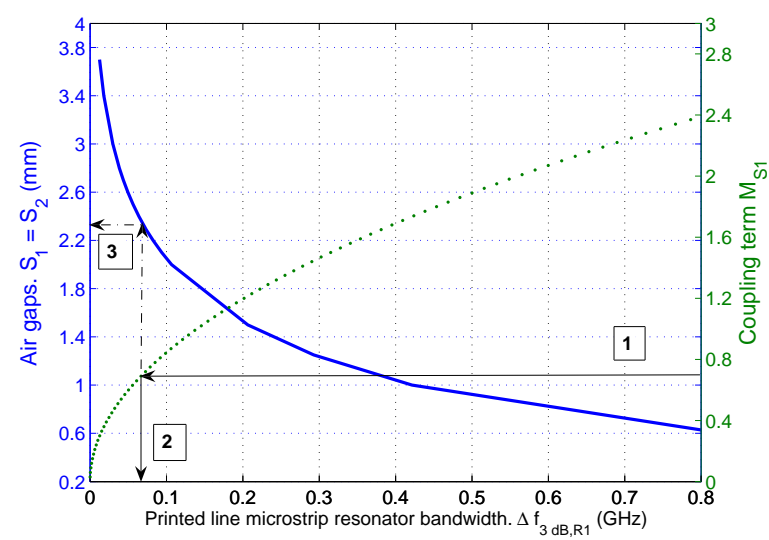

Figure 6: Printed line microstrip resonator bandwidth as a function of the air gaps (left $y$-axis), and of the coupling term $M_{S 1}$ (right $y$-axis).

resonator as a function of the air gaps $\left(S_{1}=S_{2}\right)$. On the right $y$-axis we also show the relation between the coupling term $M_{S 1}$, and the required bandwidth. Using first the right $y$-axis of the graphic, we compute the required $3 \mathrm{~dB}$ bandwidth needed to synthesize the coupling $M_{S 1}=0.7259$ (see labels 1 and 2 in Fig. 6). In this way we obtain the bandwidth $\Delta f_{3 d B, R 1}=73.765 \mathrm{MHz}$. Using now the left $y$-axis of the graphic, we obtain the required coupling gaps to synthesize this bandwidth. The air gaps are found to be $S_{1}=S_{2}=2.27 \mathrm{~mm}$.

With all these dimensions, the response of the designed printed line resonator can be compared to the ideal model provided by the coupling matrix $M_{R 1}$. It can be concluded that the dimensions of the resonator are correctly synthesized to recover the ideal response.

At this point, each one of the resonators have been individually synthesized. The last step is to put together all the elements to obtain the final response. When this is done, the cavity resonance is slightly affected by the presence of the printed line resonator. Also, the presence of the cavity walls slightly affects the behavior of the printed resonator. This mutual influence between the two elements 
is small, but requires a final fine tunning of the characteristics of the filter, as indicated in the flow chart shown in Fig. 2. In this example, the passband is perfectly recovered by adjusting the printed resonator length from $L_{r}=28.73 \mathrm{~mm}$ to $L_{r}=28.69 \mathrm{~mm}$. This represents a relative error of $0.14 \%$ in the resonator length, showing that the mutual influence between both elements of the filter is very small.

Comparing the frequency response of the whole filtering structure whose final dimensions are those collected in Table 4 (second column), and the ideal response produced by the original coupling matrix $M_{a}$, it is observed that the response inside the passband is very close to the target specifications. However, the transmission zeros are not placed at the specified frequencies. If we synthesize the coupling matrix of the obtained response, following the procedure presented in [1], the $N+2$ transversal coupling matrix results to be:

$$
M_{b}=\left(\begin{array}{rrrr}
0 & 0.7139 & -0.7449 & 0.0100 \\
0.7139 & -1.2963 & 0 & 0.7139 \\
-0.7449 & 0 & 1.2809 & 0.7449 \\
0.0100 & 0.7139 & 0.7449 & 0
\end{array}\right)
$$

It can be observed that the direct coupling term of the filtering structure after the first iteration, results to be $M_{S L}=0.01$, which is bigger than the required direct coupling term of the coupling matrix $M_{a}$ $\left(M_{S L}=0.0083\right)$. As indicated in Fig. 2, if the direct coupling term is not correctly synthesized after one iteration, the heights of the air and of the dielectric substrate $\left(L_{1}\right.$ and $\left.L_{2}\right)$ can be used to change this direct coupling. Consequently, we can select other values for the heights $L_{1}$ and $L_{2}$, and then repeat the design process from the adjustment of $L_{i n}=L_{\text {out }}$ until the end (recursive part of Fig. 2).

If we select a longer air height $\left(L_{1}\right)$, the two transmission zeros will tend to shift to higher frequencies and, simultaneously, they will tend to be more separated in frequency, thus allowing to synthesize a smaller direct coupling term, as required. For the second iteration we select an air height of $L_{1}=4 \mathrm{~mm}$. After repeating the design process we obtain the final dimensions shown in Table 4 (last column). In Fig. 7 we present the obtained frequency response of the filter, together with the target response.

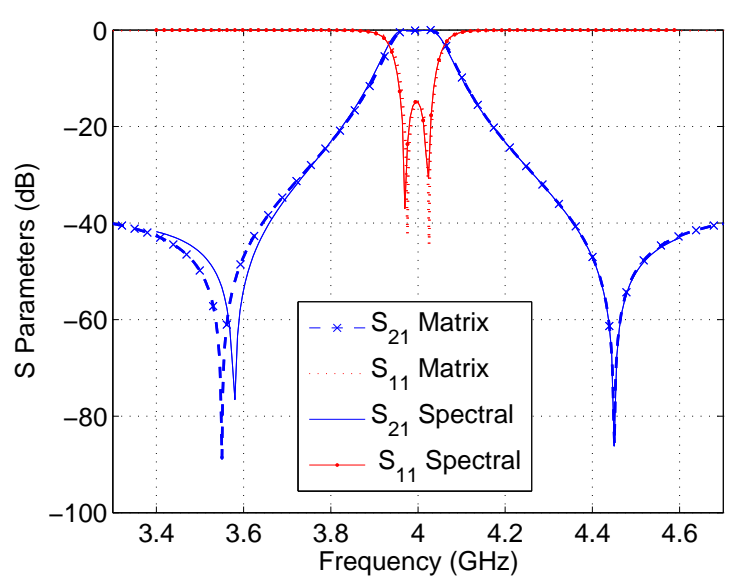

Figure 7: Ideal model $\left(M_{a}\right)$ versus simulated results for the final designed filter. Simulated results are obtained using the full wave spectral domain integral equation technique presented in [14]. Second iteration: $L_{2}=2.7 \mathrm{~mm}$ and $L_{1}=4 \mathrm{~mm}$. 
Table 4: Dimensions of the designed filter after first iteration (second column) and after final iteration (third column).

\begin{tabular}{|c|c|c|}
\hline & First iteration & Final iteration \\
\hline$L_{1}$ & $2.80 \mathrm{~mm}$ & $4.00 \mathrm{~mm}$ \\
\hline$L_{2}$ & $2.70 \mathrm{~mm}$ & $2.70 \mathrm{~mm}$ \\
\hline$L_{\text {in }}=L_{\text {out }}$ & $16.20 \mathrm{~mm}$ & $16.20 \mathrm{~mm}$ \\
\hline$b$ & $57.70 \mathrm{~mm}$ & $63.64 \mathrm{~mm}$ \\
\hline$L_{r}$ & $28.69 \mathrm{~mm}$ & $28.25 \mathrm{~mm}$ \\
\hline$S_{1}=S_{2}$ & $2.27 \mathrm{~mm}$ & $2.70 \mathrm{~mm}$ \\
\hline
\end{tabular}

This time the agreement between the designed filter and the target response is good. Therefore, as indicated in Fig. 2, the iterative algorithm can finish.

Following the novel systematic algorithm, a different example has been designed. This second example consists of a filter with two transmission zeros placed on the right side of the passband. The filter specifications are listed in Table 5. The direct synthesis procedure of [1], leads in this case to

Table 5: Filter specifications. Second design

\begin{tabular}{|c|c|}
\hline Centre frequency & $4.435 \mathrm{GHz}$ \\
\hline Bandwidth $\Delta x$ & $110 \mathrm{MHz}$ \\
\hline Return Loss & $23 \mathrm{~dB}$ \\
\hline Transmission Zeros & $4.8 \mathrm{GHz} \& 5.8 \mathrm{GHz}$ \\
\hline
\end{tabular}

the following $(N+2)$ transversal coupling matrix:

$$
M_{b}=\left(\begin{array}{rrrr}
0 & -0.7763 & 1.1472 & 0.0254 \\
-0.7763 & -1.9365 & 0 & 0.7763 \\
1.1472 & 0 & 1.7985 & 1.1472 \\
0.0254 & 0.7763 & 1.1472 & 0
\end{array}\right)
$$

In this design, the resonator $(i=1)$ with higher resonant frequency will be implemented by the cavity mode, since the resonator that changes sign is tuned above the center frequency of the filter ( $M_{11}$ is negative and $\left.M_{S 1}=-M_{L 1}\right)$. Therefore, the resonator $(i=2)$ with lower resonant frequency will be implemented by the printed line microstrip resonator.

By applying equations (3) and (14), the resonant frequency and the $3 \mathrm{~dB}$ bandwidth in the final transformed frequency axis for each resonator can be calculated. These four parameters result to be:

$$
\begin{aligned}
& f_{R 1}=4.5428 \mathrm{GHz}, \quad \Delta \mathrm{f}_{3 \mathrm{~dB}, \mathrm{R} 1}=132.56 \mathrm{MHz} \\
& f_{R 2}=4.3372 \mathrm{GHz}, \quad \Delta \mathrm{f}_{3 \mathrm{~dB}, \mathrm{R} 2}=289.53 \mathrm{MHz}
\end{aligned}
$$

With these calculations, the systematic algorithm can start. First, we fix the initial values $L_{1}=3.7$ $\mathrm{mm}$ and $a=40 \mathrm{~mm}$. The dielectric thickness is fixed to $L_{2}=3.14 \mathrm{~mm}$, since this value can be 
obtained in practice by piling up two RT-DUROID substrates of thickness $1.57 \mathrm{~mm}$, with a dielectric constant of $\varepsilon_{r}=2.2$. Furthermore, the width of the microstrip line will be fixed to $2.4 \mathrm{~mm}$, whereas the width of the input/output ports will be fixed to $2 \mathrm{~mm}$.

The design process follows the systematic algorithm previously detailed, and it will not be repeated here. Applying the whole design process to the structure, the dimensions after the first iteration are obtained.

In the filtering function of the designed structure, after the first iteration, it is observed that the transmission zeros are not placed at the right frequencies, since the direct coupling $\left(M_{S L}=0.0254\right)$ term has not been synthesized yet. Even so, the response inside the passband is very similar to the specified response provided by the coupling matrix $M_{b}$. In order to synthesize the direct coupling term, the heights of the air and of the dielectric substrate $\left(L_{1}\right.$ and $\left.L_{2}\right)$ can be adjusted. However, in this example we will maintain the value of the dielectric thickness constant, in view of doing a possible manufacturing task.

After repeating the design process with different values of the height of the air, we have found that the required height to fulfill the filter specifications is $L_{1}=4.1 \mathrm{~mm}$. The response of the final designed filter and the target response shows very good agreement, indicating that the design process has been successful, and therefore, the algorithm can finish. The designed filter has been manufactured. The prototype is shown in Fig. 8, where the target response is compared with simulated results and also with the measured response of the structure. In the simulation, losses are included in the dielectric substrate $(\tan (\delta)=0.003)$, and in the printed metallizations $\left(\sigma=3 \cdot 10^{7} \Omega^{-1} / \mathrm{m}\right)$. Good agreement is observed among target, measured and expected responses. The obtained dimensions for the final structure are also included in caption of Fig. 8.

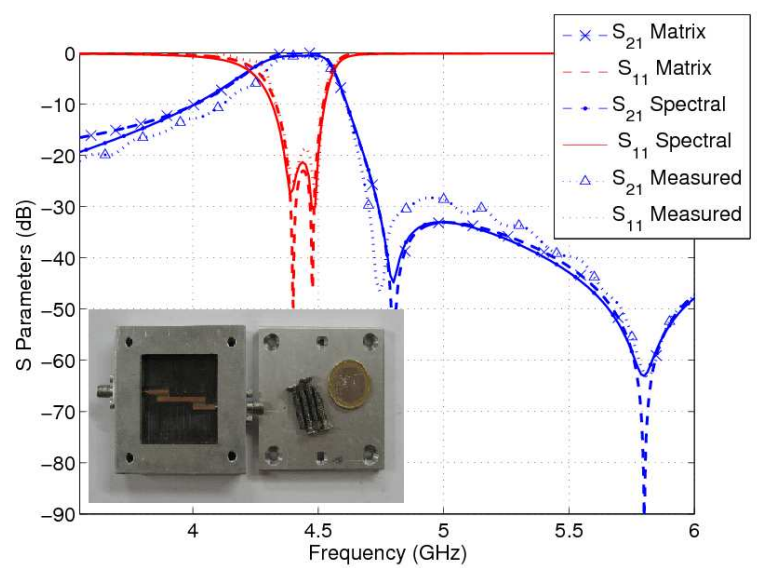

Figure 8: Comparison between target response, simulated results with losses $(\tan (\delta)=$ $\left.0.003, \sigma=3 \cdot 10^{7} \Omega^{-1} / \mathrm{m}\right)$, and measured results. Simulated results are obtained using the full wave spectral domain integral equation technique presented in [14]. Photograph of the fabricated prototype is also included. Dimensions: $L_{1}=4.10$ $\mathrm{mm}, L_{2}=3.14 \mathrm{~mm}, L_{\text {in }}=L_{\text {out }}=14.00 \mathrm{~mm}, b=41.46 \mathrm{~mm}, L_{r}=24.96 \mathrm{~mm}$ and $S_{1}=S_{2}=1.40 \mathrm{~mm}$. 


\section{Conclusions}

In this paper, a new algorithm to design transversal filters in hybrid waveguide-microstrip technology has been proposed. The technique is based on isolating each resonator from the whole structure. The dimensions of the resonators are then optimized separately. An iterative procedure has been proposed in order to adjust the final position of the transmission zeros. The basic stages of the algorithm have been illustrated by means of a design example with two transmission zeros placed symmetrically with respect to the passband. The design and manufacture of a different second order bandpass filter with two transmission zeros placed above the passband, demonstrate the validity of the proposed technique.

\section{References}

[1] R. J. Cameron, "Advanced coupling matrix synthesis techniques for microwave filters," IEEE Transactions on Microwave Theory and Techniques, vol. 51, no. 1, pp. 1-10, January 2003.

[2] S. Amari and U. Rosenberg, "Synthesis and design of novel in-line filters with one or two real transmission zeros," IEEE Transactions on Microwave Theory and Techniques, vol. 52, no. 5, pp. 1464-1478, May 2004.

[3] M. Guglielmi, P. Jarry, E. Kerherve, O. Roquebrun, and D. Schmitt, "A new family of allinductive dual-mode filters," IEEE Transactions on Microwave Theory and Techniques, vol. 49, no. 10, pp. 1764-1769, October 2001.

[4] D. C. Rebenaque, F. Q. Pereira, J. L. G. Tornero, J. P. Garcia, and A. A. Melcon, "Two simple implementations of transversal filters with coupling between non-resonant nodes," in IEEE International Microwave Symposium Digest, Long Beach, California, USA, June 2005.

[5] M. Martínez-Mendoza, J. S. Gómez-Díaz, D. C. Rebenaque, J. L. Gómez-Tornero, and A. AlvarezMelcon, "Design of a bandpass transversal filter employing a novel hybrid printed structure," in IEEE International Microwave Symposium Digest, Honolulu, Hawai, USA, 6-11 June 2007.

[6] M. M. Mendoza, J. S. G. Diaz, D. C. Rebenaque, J. L. G. Tornero, and A. A. Melcon, "Design of bandpass transversal filters employing a novel hybrid structure," IEEE Transactions on Microwave Theory and Techniques, vol. 55, no. 12, pp. 2670-2678, December 2007.

[7] J. W. Bandler, W. Kellermann, and K. Madsen, "A superlinearly convergent minimax algorithm for microwave circuit design," IEEE Transactions on Microwave Theory and Techniques, vol. 33, no. 12, pp. 1519-1530, December 1985.

[8] J. W. Bandler, R. M. Biernacki, S. H. Chen, P. A. Grobelny, and R. H. Hemmers, "Space mapping technique for electromagnetic optimization," IEEE Transactions on Microwave Theory and Techniques, vol. 42, no. 12, pp. 2536-2544, December 1994.

[9] J. P. Garcia, F. Q. Pereira, D. C. Rebenaque, J. G. Tornero, and A. A. Melcon, "A multilayered shielded microwave circuits design method based on genetic algorithms and neural networks," in IEEE, MTT-S International Microwave Symposium, San Francisco, CA, USA, 11-16 June 2006, pp. 1427-1430, no. TH1F-02. 
[10] M. Guglielmi, "A simple CAD procedure for microwave filters and multiplexers," IEEE Transactions on Microwave Theory and Techniques, vol. 42, no. 7, pp. 1347-1352, July 1994.

[11] J.-S. Hong and M. J. Lancaster, Microstrip Filters for RF/Microwave Applications. John Wiley sons, 2001.

[12] G. Matthaei and E. Yones, Microwave Filters, Impedance Matching Networks, and Coupling Structures. Boston, Massachusetts, USA: Artech House, 1980.

[13] S. Amari and U. Rosenberg, "A universal building block for advanced modular design of microwave filters," IEEE Microwave and Wireless Components Letters, vol. 13, no. 12, pp. 541-543, December 2003.

[14] A. A. Melcon, J. R. Mosig, and M. Guglielmi, "Efficient CAD of boxed microwave circuits based on arbitrary rectangular elements," IEEE Transactions on Microwave Theory and Techniques, vol. 47, no. 7, pp. 1045-1058, July 1999. 